\title{
Electronic structure and band alignment of zinc nitride, $\mathrm{Zn}_{3} \mathrm{~N}_{\mathbf{2}}$
}

Cite this: RSC Adv., 2014, 4, 3306

\author{
Su-Hyun Yoo, ${ }^{a}$ Aron Walsh, ${ }^{* b}$ David O. Scanlon ${ }^{c}$ and Aloysius Soon ${ }^{\star a d}$
}

Received 11th November 2013

Accepted 3rd December 2013

DOI: $10.1039 / c 3 r a 46558 f$

www.rsc.org/advances

Zinc nitride $\left(\mathrm{Zn}_{3} \mathrm{~N}_{2}\right)$ is a promising candidate for optoelectronics applications due to its high electron mobility and high electrical conductivity. It is also thought that $\mathrm{Zn}_{3} \mathrm{~N}_{2}$ can be used as a starting material to achieve $\mathrm{p}$-type conductivity in $\mathrm{ZnO}$-based oxide homojunctions. In this work, the electronic structure of bulk $\mathrm{Zn}_{3} \mathrm{~N}_{2}$ is studied using density-functional theory (DFT) with different approximations to the exchange-correlation functional, ranging from (semi-)local functionals to the quasiparticle $G_{0} W_{0}$ approach. We predict a bandgap in the range of $0.9-1.2 \mathrm{eV}$, reconciling the scattered values reported in experiments, and a remarkably low work function (ionisation potential) of $4.2 \mathrm{eV}$ for the (111) surface.

\section{Introduction}

For optoelectronic applications such as light emitting diodes (LEDs) and laser diodes, all-oxide homojunctions are very much desired and have distinct advantages over their hetero-counterparts, such as the prevention of heterojunction interfacial mismatch (resulting in lower efficiency), as well as reducing the high cost involved in the fabrication of semiconductor heterojunctions.

In this aspect, zinc oxide ( $\mathrm{ZnO})$ is often considered as the choice material for the making of the oxide $\mathrm{p}-\mathrm{n}$ homojunction, especially for ultraviolet UV/blue optoelectronic applications due to its wide bandgap energy of $3.437 \mathrm{eV}$ (at $2 \mathrm{~K}$ ) and large exciton binding energy, $60 \mathrm{meV} .^{1}$ Unfortunately, due to the fact that the $\mathrm{ZnO}$ exhibits naturally strong $\mathrm{n}$-type conductivity under the influence of native defects or adventitious donors, alternative ways to achieve p-type conductivity in $\mathrm{ZnO}$ are currently pursued by many researchers hoping to understand the

${ }^{a}$ Global E3 Institute, Department of Materials Science and Engineering, Yonsei University, Seoul 120-749, Korea. E-mail: aloysius.soon@yonsei.ac.kr

${ }^{b}$ Centre for Sustainable Chemical Technologies, Department of Chemistry, University of Bath, Claverton Down, Bath BA2 7AY, UK. E-mail: a.walsh@bath.ac.uk

${ }^{'}$ Kathleen Lonsdale Materials Chemistry, Department of Chemistry, University College London, 20 Gordon Street, London WC1H OAJ, UK

${ }^{d}$ School of Physics, The University of Sydney, NSW 2006, Australia fundamental physics to design and achieve robust p-type conductivity in $\mathrm{ZnO}^{2-5}$

Recently, it has been proposed that a promising route to fabricate $\mathrm{ZnO} \mathrm{p}-\mathrm{n}$ homojunctions could be the transformation of zinc nitride $\left(\mathrm{Zn}_{3} \mathrm{~N}_{2}\right)$ into p-type $\mathrm{ZnO}$ by thermal treatment in oxidizing ambient conditions. ${ }^{6-8}$ The interest in $\mathrm{Zn}_{3} \mathrm{~N}_{2}$ is also due to its intrinsic high electron mobility of 100 $\mathrm{cm}^{2} \mathrm{~V}^{-1} \mathrm{~s}^{-1}$ and as well as a high carrier concentration, $10^{20}$ $\mathrm{cm}^{-3}{ }^{9}$ The low cost involved in the production of $\mathrm{Zn}_{3} \mathrm{~N}_{2}$ also makes it an ideal choice for mass production in electronic systems. Both the binary zinc nitride system and the ternary zinc oxynitride mixture are composed of earth abundant elements, which makes them of further interest for solar energy harvesting. Control of the optical response, by varying the composition, could offer a route to low-cost, high-efficiency solar cells.

Unfortunately, as compared to $\mathrm{ZnO}$, the fundamental bulk, surface and interface properties of $\mathrm{Zn}_{3} \mathrm{~N}_{2}$ are poorly understood, possibly be due to the fact that $\mathrm{Zn}_{3} \mathrm{~N}_{2}$ oxidizes rather easily under ambient conditions. Given the difficulty to study the properties of pristine $\mathrm{Zn}_{3} \mathrm{~N}_{2}$, the true value of its intrinsic bandgap energy is still a matter of controversy, and reported values range from 1.0 to $3.2 \mathrm{eV}^{.9-13}$ This current lack of understanding clearly hinders the further development of ZnO-based $\mathrm{p}-\mathrm{n}$ homojunctions in devices.

In this letter, using density-functional theory (DFT), we will show that choice of the exchange-correlation $(x c)$ functional is crucial in providing a consistent description of the electronic structure of $\mathrm{Zn}_{3} \mathrm{~N}_{2}$. Semi-local functionals severely underestimate the bandgap energy and augmenting them with the Hubbard $U$ model does not improve this shortcoming, while hybrid density-functionals provide the most consistent picture when compared to the many-body $G W$ theory.

\section{Computational approach}

All calculations were performed using the Vienna $A b$ initio Simulations Package (VASP) code, ${ }^{14,15}$ employing a planewave 
basis set with an energy cutoff of $500 \mathrm{eV}$, within the projector augmented-wave (PAW) approach. ${ }^{16}$ In this work, the 40 -atom primitive cell of $\mathrm{Zn}_{3} \mathrm{~N}_{2}$ was used for all calculations, and the Brillouin-zone integrations were performed using a gammacentered k-point grid of $4 \times 4 \times 4$. The atomic geometry and electronic structure of bulk $\mathrm{Zn}_{3} \mathrm{~N}_{2}$ was first calculated using the local-density approximation (LDA), ${ }^{17}$ the generalized gradient approximation (GGA) due to Perdew, Burke, and Ernzerhof (PBE), ${ }^{\mathbf{1 8 , 1 9}}$ as well as the Hubbard $U$ augmented forms of these approximations. For the LDA/GGA $+U$ approach, various effective $U$ values on the $\mathrm{Zn} 3 \mathrm{~d}$ states were tested (ranging from 1 to $7 \mathrm{eV}$ ), and an optimal $U$ of $5 \mathrm{eV}$ was chosen. (See Appendix for more information) To further assess the accuracy of the electronic structure of bulk $\mathrm{Zn}_{3} \mathrm{~N}_{2}$ within (semi-)local approximations, we have also performed electronic structure calculations using the modified Becke-Johnson meta-GGA ${ }^{20}$ due to Tran and Blaha (TB-mBJ), ${ }^{21}$ the HSE06 $^{22,23}$ and PBE0 ${ }^{24}$ hybrid functionals, as well as the perturbative quasiparticle $G_{0} W_{0}^{25,26}$ approach performed on top of the HSE06 wavefunction. ${ }^{27}$

\section{Results and discussion}

$\mathrm{Zn}_{3} \mathrm{~N}_{2}$ is known to crystalize in the anti-bixbyite structure (space group of $I a \overline{3}$ ), with a body-centered cubic lattice. The conventional cell of $\mathrm{Zn}_{3} \mathrm{~N}_{2}$ has 80 atoms, as illustrated in Fig. 1a. The anti-bixbyite structure can be thought of as the calcium fluorite $\left(\mathrm{CaF}_{2}\right)$ structure with $\mathrm{N}$ atoms occupying the cation sites, while the $\mathrm{Zn}$ atoms are located at three quarters of the anion sites. In this structure, the $\mathrm{N}$ atoms are coordinated to 6 neighbouring $\mathrm{Zn}$ atoms at 2 distinct sites represented by the $8 \mathrm{~b}(1 / 4,1 / 4,1 / 4)$ and $24 \mathrm{~d}(x, 0,1 / 4)$ Wyckoff positions, as illustrated in Fig. 1b.

Table 1 contains the calculated equilibrium lattice constant (neglecting zero-point vibrations) and the direct bandgap energy of $\mathrm{Zn}_{3} \mathrm{~N}_{2}$ using various levels of approximations to the exchange-correlation density functional, as well as $G W$ theory, in comparison to available experimental values.
Table 1 Calculated equilibrium lattice constant, $a$ and the direct bandgap energy, $E_{\mathrm{g}}$ of $\mathrm{Zn}_{3} \mathrm{~N}_{2}$, using various levels of approximations. The corresponding experimental values are reported in the last row

\begin{tabular}{lll}
\hline & $a(\AA)$ & $E_{\mathrm{g}}(\mathrm{eV})$ \\
\hline LDA & 9.593 & 0.03 \\
PBE & 9.857 & No gap \\
LDA $+U$ & 9.418 & 0.62 \\
PBE $+U$ & 9.692 & 0.23 \\
TB-mBJ & - & 0.95 \\
HSE06 & 9.756 & 0.86 \\
PBE0 & 9.767 & 1.48 \\
$G_{0} W_{0}$ & - & 1.15 \\
Experiment & $9.769^{28}$ & $1.0-3.2^{9-13}$
\end{tabular}

The structural parameters calculated using PBE $+U(9.692 \AA)$, PBE0 (9.767 ̊) and HSE06 (9.756 ̊) provide the best agreement with the reported experimental value of $9.769 \AA$. The LDA and LDA $+U$ values are clearly underestimated (up to $3.6 \%$ ), while PBE overestimates it by about $1 \%$. These are generally in line with analogous studies of other nitride materials. ${ }^{29-33}$ Thus, for the TB-mBJ and $G_{0} W_{0}$ calculations, structural parameters of bulk $\mathrm{Zn}_{3} \mathrm{~N}_{2}$ were fixed to the values determined using HSE06 as those methods cannot currently be used for lattice optimisations.

Turning to the electronic structure of $\mathrm{Zn}_{3} \mathrm{~N}_{2}$, we find that the values of the bandgap energy are just as scattered as those reported from experiments. Recent studies have shown that $G_{0} W_{0}$ generally has quantitative accuracy when describing the bandgaps of most (non-transition metal) semiconductors. ${ }^{34-37}$ In this sense, the calculated $G_{0} W_{0}$ bandgap energy value will be used as the benchmark and is found to be $1.15 \mathrm{eV}$. The calculations using LDA and PBE yield virtually a zero bandgap, with their Hubbard $U$ counterparts improves it by about less than $0.5 \mathrm{eV}$, but still largely underestimated as compared to the $G_{0} W_{0}$ value. Both the HSE06 and PBE0 hybrid density-functionals further improve the bandgap energy with PBE0 overestimating it by about 0.5 eV. Interestingly, the recent meta-GGA functional, TB-mBJ (a)

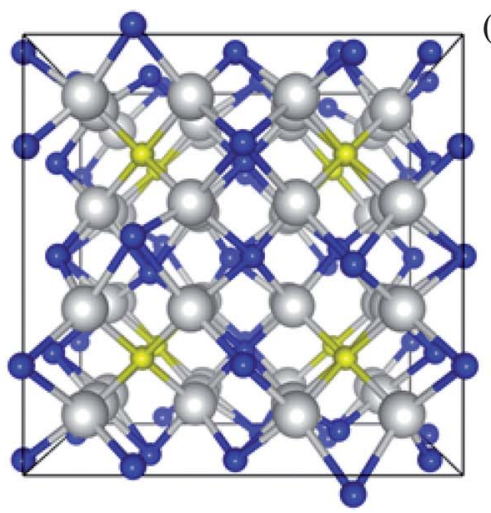

(b)

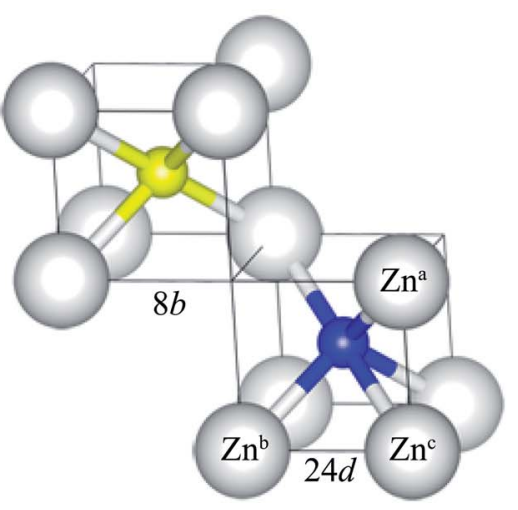

Fig. 1 (Color online) (a) Perspective view of the atomic structure of bulk zinc nitride. (b) Spatial arrangement of zinc atoms around 2 distinct types of nitrogen atoms (denoted as $8 \mathrm{~b}$ and $24 \mathrm{~d}$ Wyckoff positions). The zinc atoms are represented as large white spheres, while the nitrogen atoms ( $8 \mathrm{~b}$ and $24 \mathrm{~d}$ ) are shown as small yellow and blue spheres, respectively. 
gives a bandgap energy value of $0.95 \mathrm{eV}$ which agrees the best with the $G_{0} W_{0}$ bandgap energy value. However, the band gap is just one relative energy difference from the full band structure and this agreement might not provide a fully consistent picture to the electronic structure of bulk $\mathrm{Zn}_{3} \mathrm{~N}_{2}$. To illustrate this, we calculated and plot the electronic bandstructures of bulk $\mathrm{Zn}_{3} \mathrm{~N}_{2}$ obtained by LDA, TB-mBJ, and HSE06 in Fig. 2.

One challenge for electronic structure techniques applied to $\mathrm{ZnO}$ and related semiconductors is in the treatment of shallow core $\mathrm{d}$ states. ${ }^{38}$ Focusing on the relative position of the localized $\mathrm{Zn} 3 \mathrm{~d}$ states in the sub-valence band (near -6 to $-8 \mathrm{eV}$ ), it becomes apparent that the meta-GGA still fails to correct the level of $\mathrm{Zn} 3 \mathrm{~d}$ states as predicted by the LDA (i.e. still too high with respect to the valence band maximum), ${ }^{39,40}$ albeit bringing the bandgap energy value closest to that by the $G_{0} W_{0}$ approach..$^{41}$ On the other hand, using HSE06 which includes a percentage of the exact non-local Fock exchange, then reconciles the correct placement of the $\mathrm{Zn} 3 \mathrm{~d}$ states.

Thus, we now proceed to further study the electronic structure of bulk $\mathrm{Zn}_{3} \mathrm{~N}_{2}$ using the HSE06 density-functional with confidence that this level of theory will provide a balanced and consistent description of its electronic structure.

In Fig. 3a, we analyze the electronic density-of-states in three regions: (i) the sub-valence band ( -9 to $-6.5 \mathrm{eV}$ ), (ii) the uppervalence band $(-6.5$ to $0 \mathrm{eV})$, and (iii) the conduction band (above $0.86 \mathrm{eV}$ ). The sub-valence band finds a large contribution from the highly localized $\mathrm{Zn} 3 \mathrm{~d}$ states, while the overlapping of the $\mathrm{Zn} 3 \mathrm{~d}$ and $\mathrm{N} 2 \mathrm{p}$ states can be seen in the upper-valence band with the $\mathrm{N} 2 \mathrm{p}$ states more pronounced nearer the top of the valence band. The conduction band comprises of the empty $\mathrm{Zn}$ $4 \mathrm{~s}$ and $\mathrm{N} 2 \mathrm{~s}$ states. This corroborates well with the partial electron densities plotted for the states near the band edges in Fig. $3 \mathrm{~b}$ and $\mathrm{c}$.

Given that the physics of ZnO-based $\mathrm{p}-\mathrm{n}$ homojunctions is a major research goal, as a first-step, it will be important to
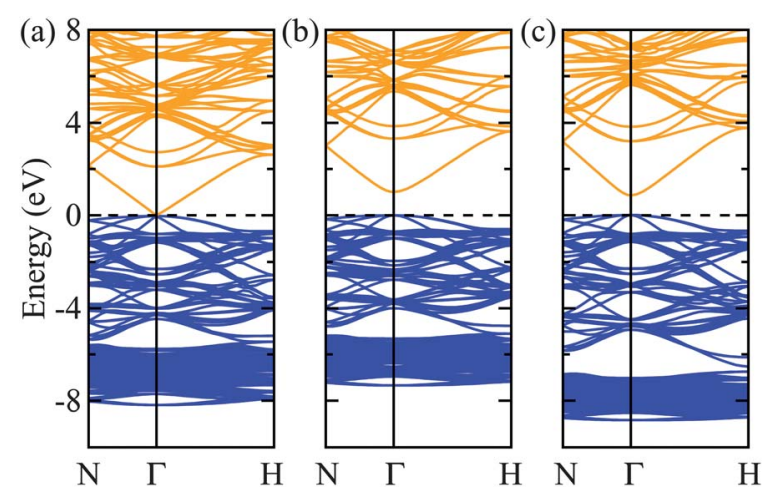

Fig. 2 (Color online) The calculated electronic band structure of $\mathrm{Zn}_{3} \mathrm{~N}_{2}$ obtained by (a) LDA, (b) TB-mBJ and (c) HSEO6 densityfunctionals. The valence band edge is set to $0 \mathrm{eV}$. The conduction and valence bands are shown as yellow and blue lines, respectively. The bandgap energies calculated by TB-mBJ and HSEO6 are 0.95 and $0.86 \mathrm{eV}$, respectively, whereas the bandgap energy by LDA is $0.03 \mathrm{eV}$.

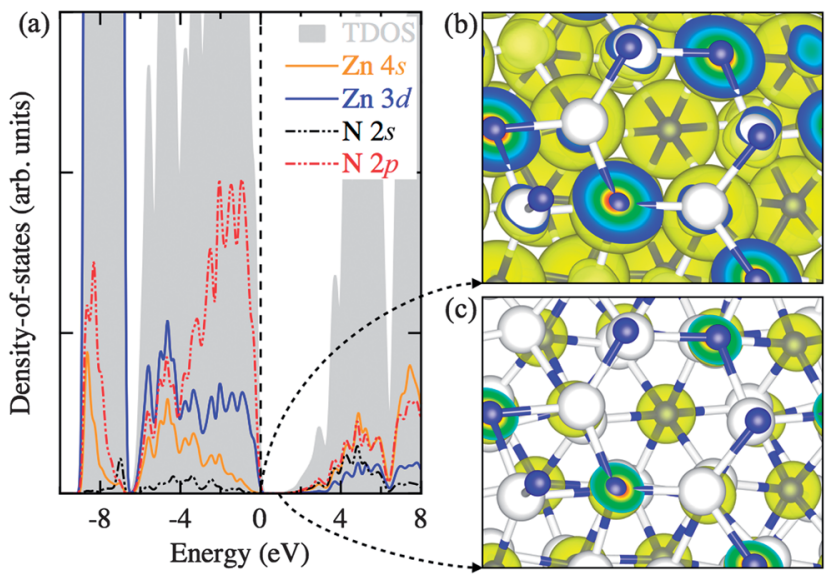

Fig. 3 (Color online) (a) Electronic density-of-states for bulk $Z n_{3} N_{2}$. (b) 3D isosurfaces of the partial electron density of the states near the valence band edge and (c) that near the conduction band edge. These are calculated using the HSEO6 density-functional. In (a), the valence band edge is set to $0 \mathrm{eV}$. The continuous lines indicate the contribution of $\mathrm{Zn} 3 \mathrm{~d}$ (in blue) and $4 \mathrm{~s}$ (in orange) states while the dashed lines represents the states of $N 2 p$ (in red) and $2 s$ (in black). In (b) and (c), the $\mathrm{Zn}$ atoms are represented as large white spheres while the $\mathrm{N}$ atoms are shown as small blue spheres, respectively.

understand the relative band alignment of pristine $\mathrm{ZnO}$ and $\mathrm{Zn}_{3} \mathrm{~N}_{2}$. In Fig. 4, we plot the natural valence band alignment based on our calculated ionisation potential of $\mathrm{Zn}_{3} \mathrm{~N}_{2}$ and the previously calculated value for $\mathrm{ZnO}$ using the quantum mechanics/molecular mechanics (QM/MM) approach..$^{42}$ This $\mathrm{QM} / \mathrm{MM}$ approach has been shown to produce very accurate ionization potentials for materials, as it has direct access to the vacuum level. ${ }^{43}$ We calculated the ionisation potential for $\mathrm{Zn}_{3} \mathrm{~N}_{2}$ using the slab model, adopting the standard approach as indicated by Van de Walle et al. ${ }^{44}$ First, a surface work function

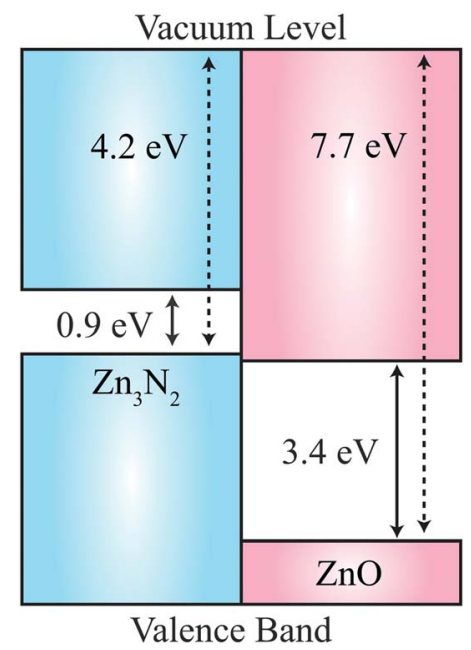

Fig. 4 (Color online) Predicted band alignment between $Z n_{3} N_{2}$ and $\mathrm{ZnO}$. The calculated work functions (ionization potentials) of $\mathrm{Zn}_{3} \mathrm{~N}_{2}$ (HSE06) and $\mathrm{ZnO}$ (B97-1) are 4.2 and $7.7 \mathrm{eV}$, respectively, with their corresponding bandgap energies being 0.9 and $3.4 \mathrm{eV}$. 
Table 2 Structural properties (in $\AA$ ) of $Z n_{3} N_{2}$ obtained by PBE $+U$ functional. Lattice constant and bond lengths between nitrogen and zinc atom. $\mathrm{N} 1$ is nitrogen atom located at $8 \mathrm{~b}$ Wyckoff position, N2 located at $24 \mathrm{~d} . \mathrm{Zn}^{a}, \mathrm{Zn}^{b}$ and $\mathrm{Zn}^{c}$ are the neighbor atoms of N2 atom. Errors with experimental results are given in parentheses (in \%)

\begin{tabular}{|c|c|c|c|c|c|c|}
\hline Functional & $U_{\text {eff }}($ in eV) & Lattice constant & $\mathrm{Zn}-\mathrm{N} 1$ & $\mathrm{Zn}^{a}-\mathrm{N} 2$ & $\mathrm{Zn}^{b}-\mathrm{N} 2$ & $\mathrm{Zn}^{c}-\mathrm{N} 2$ \\
\hline \multirow[t]{16}{*}{$\mathrm{PBE}+U$} & \multirow[t]{2}{*}{0} & 9.857 & 2.144 & 2.015 & 2.065 & 2.329 \\
\hline & & $(0.90)$ & $(0.52)$ & $(0.95)$ & $(-0.15)$ & $(2.96)$ \\
\hline & \multirow[t]{2}{*}{1} & 9.830 & 2.137 & 2.011 & 2.065 & 2.312 \\
\hline & & $(0.62)$ & $(0.19)$ & $(0.75)$ & $(-0.15)$ & $(2.21)$ \\
\hline & \multirow[t]{2}{*}{2} & 9.800 & 2.129 & 2.007 & 2.063 & 2.296 \\
\hline & & $(0.32)$ & $(-0.19)$ & $(0.55)$ & $(-0.24)$ & $(1.50)$ \\
\hline & \multirow[t]{2}{*}{3} & 9.768 & 2.121 & 2.002 & 2.061 & 2.278 \\
\hline & & $(-0.01)$ & $(-0.56)$ & $(0.30)$ & $(-0.34)$ & $(0.71)$ \\
\hline & \multirow[t]{2}{*}{4} & 9.733 & 2.112 & 1.997 & 2.058 & 2.262 \\
\hline & & $(-0.37)$ & $(-0.98)$ & $(0.05)$ & $(-0.48)$ & $(0.00)$ \\
\hline & \multirow[t]{2}{*}{5} & 9.695 & 2.103 & 1.991 & 2.054 & 2.244 \\
\hline & & $(-0.76)$ & $(-1.41)$ & $(-0.25)$ & $(-0.68)$ & $(-0.80)$ \\
\hline & \multirow[t]{2}{*}{6} & 9.653 & 2.093 & 1.984 & 2.049 & 2.227 \\
\hline & & $(-1.19)$ & $(-1.88)$ & $(-0.60)$ & $(-0.92)$ & $(-1.55)$ \\
\hline & \multirow[t]{2}{*}{7} & 9.605 & 2.081 & 1.975 & 2.043 & 2.209 \\
\hline & & $(-1.68)$ & $(-2.44)$ & $(-1.05)$ & $(-1.21)$ & $(-2.34)$ \\
\hline \multicolumn{2}{|l|}{ Exp. $^{28}$} & 9.769 & 2.133 & 1.996 & 2.068 & 2.262 \\
\hline
\end{tabular}

is determined by aligning the electronic band structure with respect to the vacuum level determined by a slab calculation of the stoichiometric (111) surface termination, which has no residual dipoles. The slab consisted of four quadrupolar layers (160 atoms). The natural band offset is thus the offset of the bulk ionisation potentials for each material, relative to the vacuum level. This alignment is free of any strain affects experienced at a true interface, and so represents a "natural band alignment" that would be seen between two unstrained materials.

The value of the HSE06-derived work function of $\mathrm{Zn}_{3} \mathrm{~N}_{2}$ is $4.2 \mathrm{eV}$, which is aligned to the bulk ionisation potential using the core $\mathrm{N} 2$ p core level in the centre of the slab. The work function is remarkably low, e.g. for the photocatalytic oxide $\mathrm{TiO}_{2}$ has a value close to $8 \mathrm{eV}^{43}$ The natural value of $4.2 \mathrm{eV}$, places the valence band of $\mathrm{Zn}_{3} \mathrm{~N}_{2} 3.5 \mathrm{eV}$ above that of $\mathrm{ZnO}$, which results in a Type-IIB (misaligned) band offset. ${ }^{45}$ While it is generally expected that the valence band of the nitride would be higher in energy than an oxide due to the relative binding energy of the $\mathrm{N}$ $2 p$ and $\mathrm{O} 2 \mathrm{p}$ atomic energy levels, this effect is exaggerated due to unusual octahedral coordination of $\mathrm{N}$ in $\mathrm{Zn}_{3} \mathrm{~N}_{2}$ in contrast to the regular tetrahedral environments found for III-V semiconductors. The value is comparable to the new class of nitride electrode materials, which have work functions as low as 2.6 $\mathrm{eV}^{46,47} \mathrm{We}$ emphasise that the predicted natural band offset is representative of the two isolated systems, while for a synthesised heterostructure, interfacial effects (structural, compositional and electrostatic changes) could alter the Type-IIB behaviour.

\section{Conclusions}

In summary, we studied the electronic structure of bulk $\mathrm{Zn}_{3} \mathrm{~N}_{2}$ systematically from the LDA to the many-body $G W$ approach.
We find that (semi-)local density-functionals (including the recent TB-mBJ functional) do not provide a consistent description of the electronic structure of bulk $\mathrm{Zn}_{3} \mathrm{~N}_{2}$. The hybrid density-functional HSE06 provides the most consistent picture, yielding a bandgap energy value of $1 \mathrm{eV}$, close to that predicted by the $G_{0} W_{0}$ approach. The predicted work function of $\mathrm{Zn}_{3} \mathrm{~N}_{2}$ is remarkably low, which is closer to alkaline earth nitrides than typical III-V materials. Future work should consider the interfacial structure of a $\mathrm{ZnO} / \mathrm{Zn}_{3} \mathrm{~N}_{2}$ composite and the effects of partial oxidation on the electronic structure.

\section{Appendix}

Here we report the structural (in Table 2) and electronic properties (in Fig. 5) of $\mathrm{Zn}_{3} \mathrm{~N}_{2}$, as calculated using the PBE $+U$ approach. We tested different $U_{\text {eff }}$ values, ranging from 0 to 7 $\mathrm{eV}$, and benchmarked our results with that calculated using the HSE06 hybrid DFT functional for the electronic structure and available experimental results for structural properties.

\section{Acknowledgements}

We acknowledge membership of the UK's HPC Materials Chemistry Consortium, which is funded by the EPSRC Grant EP/F067496, and the Materials Design Network. Computational resources have also been provided by the KISTI supercomputing center (KSC-2012-C2-28, KSC-2013-C2-022). D.O.S. is grateful to the Ramsay Memorial Trust and the University College London for the provision of a Ramsay Fellowship. A.W. acknowledges support from the Royal Society. S.H.Y. and A.S. are supported by the National Research Foundation of Korea (NRF Grant no. 2011- 0013201) and the Global Frontier R\&D Program (2013073298) by the Ministry of Science, ICT \& Future Planning, Korea. 

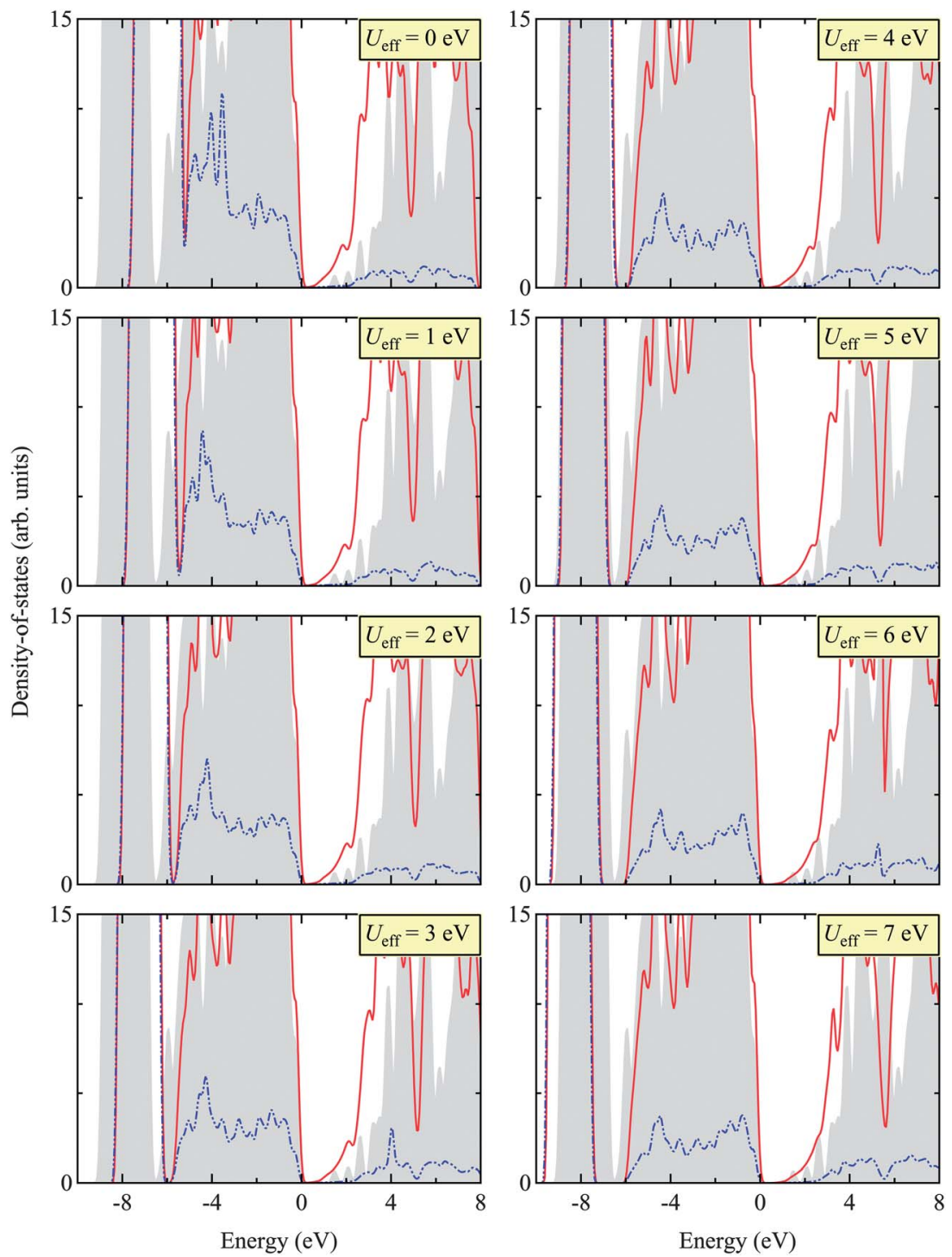

Fig. 5 (Color online) Total (TDOS) and projected (PDOS) density of states for $\mathrm{Zn}_{3} \mathrm{~N}_{2}$ calculated by HSE06 (as a benchmark) and PBE $+U$ with different $U_{\text {eff }}$ values (ranging from 0 to $7 \mathrm{eV}$ ). The gray shade indicates TDOS obtained using the HSE06 functional, the red line TDOS using the PBE $+U$ approach, and the blue dotted line PDOS of $\mathrm{Zn} 3 \mathrm{~d}$ states using the PBE $+U$ approach. The valence band edge is set to 0 eV. The appropriate $U_{\text {eff }}$ is then determined as $5 \mathrm{eV}$, matching the sub-valence band (near $-8 \mathrm{eV}$ ) of HSE06 and PBE $+U$. We find this value of $U_{\text {eff }}=5 \mathrm{eV}$ in close agreement with other $\mathrm{Zn}$-based compounds, e.g. $\mathrm{ZnO} .^{48-50}$

\section{References}

1 D. C. Look, Mater. Sci. Eng., B, 2001, 80, 383.

2 C. G. V. de Walle, Phys. Rev. Lett., 2000, 85, 1012.

3 S. B. Zhang, S.-H. Wei and A. Zunger, Phys. Rev. B: Condens. Matter Mater. Phys., 2001, 63, 075205.

4 W.-J. Lee, J. Kang and K. J. Chang, Phys. Rev. B: Condens. Matter Mater. Phys., 2006, 73, 024117.

5 C. R. A. Catlow, A. A. Sokol and A. Walsh, Chem. Commun., 2011, 47, 3386.

6 D. Wang, Y. C. Liu, R. Mu, J. Y. Zhang, Y. M. Lu, D. Z. Shen and X. W. Fan, J. Phys.: Condens. Matter, 2004, 16, 4635.

7 M. Bär, K. S. Ahn, S. Shet, Y. Yan, L. Weinhardt, O. Fuchs, M. Blum, S. Pookpanratana, K. George, W. Yang, et al., Appl. Phys. Lett., 2009, 94, 012110.
8 N. H. Erdogan, K. Kara, H. Ozdamar, R. Esen and H. Kavak, Appl. Surf. Sci., 2013, 271, 70.

9 M. Futsuhara, K. Yoshioka and O. Takai, Thin Solid Films, 1998, 322, 274.

10 K. Kuriyama, Y. Takahashi and F. Sunohara, Phys. Rev. B: Condens. Matter Mater. Phys., 1993, 48, 2781.

11 K. Toyoura, H. Tsujimura, T. Goto, K. Hachiya, R. Hagiwara and Y. Ito, Thin Solid Films, 2005, 492, 88.

12 T. Suda and K. Kakishita, J. Appl. Phys., 2006, 99, 076101.

13 G. Z. Xing, D. D. Wang, B. Yao, L. F. N. A. Qune, T. Yang, Q. He,

J. H. Yang and L. L. Yang, J. Appl. Phys., 2010, 108, 083710.

14 G. Kresse and J. Hafner, Phys. Rev. B: Condens. Matter Mater. Phys., 1993, 47, 558.

15 G. Kresse and J. Furthmüller, Phys. Rev. B: Condens. Matter Mater. Phys., 1996, 54, 11169. 
16 G. Kresse and D. Joubert, Phys. Rev. B: Condens. Matter Mater. Phys., 1999, 59, 1758.

17 J. P. Perdew and A. Zunger, Phys. Rev. B: Condens. Matter Mater. Phys., 1981, 23, 5048.

18 J. P. Perdew, K. Burke and M. Ernzerhof, Phys. Rev. Lett., 1996, 77, 3865.

19 J. P. Perdew, K. Burke and M. Ernzerhof, Phys. Rev. Lett., 1997, 78, 1396.

20 A. D. Becke and E. R. Johnson, J. Chem. Phys., 2006, 124, 221101.

21 F. Tran and P. Blaha, Phys. Rev. Lett., 2009, 102, 226401.

22 J. Heyd, G. E. Scuseria and M. Ernzerhof, J. Chem. Phys., 2003, 118, 8207.

23 J. Heyd, G. E. Scuseria and M. Ernzerhof, J. Chem. Phys., 2006, 124, 219906.

24 J. P. Perdew, M. Ernzerhof and K. Burke, J. Chem. Phys., 1996, 105, 9982.

25 L. Hedin, Phys. Rev., 1965, 139, A796.

26 M. Shishkin and G. Kresse, Phys. Rev. B: Condens. Matter Mater. Phys., 2007, 75, 235102.

27 N. Marom, F. Caruso, X. Ren, O. T. Hofmann, T. Körzdörfer, J. R. Chelikowsky, A. Rubio, M. Scheffler and P. Rinke, Phys. Rev. B: Condens. Matter Mater. Phys., 2012, 86, 245127.

28 D. E. Partin, D. J. Williams and M. O'Keeffe, J. Solid State Chem., 1997, 132, 56.

29 T. Lee, B. Delley, C. Stampfl and A. Soon, Nanoscaele, 2012, 4, 5183.

30 X. M. Duan and C. Stampfl, Phys. Rev. B: Condens. Matter Mater. Phys., 2009, 79, 035207.

31 M. Fuchs, J. L. F. D. Silva, C. Stampfl, J. Neugebauer and M. Scheffler, Phys. Rev. B: Condens. Matter Mater. Phys., 2002, 65, 245212.

32 Z. Li, P. Wang, H. Chen and X. Cheng, Physica B, 2011, 406, 1182.

33 R. Long, Y. Dai, L. Yu, M. Guo and B. Huang, J. Phys. Chem. B, 2007, 111, 3379.

34 H. Jiang, P. Rinke and M. Scheffler, Phys. Rev. B: Condens. Matter Mater. Phys., 2012, 86, 125115.
35 X.-Z. Li, R. Gómez-Abal, H. Jiang, C. Ambrosch-Draxl and M. Scheffler, 2012, 14, 023006.

36 M. A. L. Marques, J. Vidal, M. J. T. Oliveira, L. Reining and S. Botti, Phys. Rev. B: Condens. Matter Mater. Phys., 2011, 83, 035119.

37 L. A. Burton and A. Walsh, Appl. Phys. Lett., 2013, 102, 132111.

38 P. Rinke, A. Qteish, J. Neugebauer, C. Freysoldt and M. Scheffler, New J. Phys., 2005, 7, 126.

39 D. J. Singh, Phys. Rev. B: Condens. Matter Mater. Phys., 2010, 82, 205102.

40 H. Jiang, J. Chem. Phys., 2013, 138, 134115.

41 D. Waroquiers, A. Lherbier, A. Miglio, M. Stankovski, S. Poncé, M. J. T. Oliveira, M. Giantomassi, G.-M. Rignanese and X. Gonze, Phys. Rev. B: Condens. Matter Mater. Phys., 2013, 87, 075121.

42 A. A. Sokol, S. A. French, S. T. Bromley, C. R. A. Catlow, H. J. J. van Dam and P. Sherwood, Faraday Discuss., 2007, 134, 267.

43 D. O. Scanlon, C. W. Dunnill, J. Buckeridge, S. A. Shevlin, A. J. Logsdail, S. M. Woodley, C. R. A. Catlow, M. J. Powell, R. G. Palgrave, I. P. Parkin, et al., Nat. Mater., 2013, 12, 798.

44 P. G. Moses, M. Miao, Q. Yan and C. G. Van de Walle, J. Chem. Phys., 2011, 134, 084703.

45 P. Y. Yu and M. Cardona, Fundamentals of Semiconductors, Springer-Verlag, Berlin Heidelberg, 2010, vol. 4.

46 A. Walsh and D. O. Scanlon, J. Mater. Chem. C, 2013, 1, 3525.

47 K. Lee, S. W. Kim, Y. Toda, S. Matsuishi and H. Hosono, Nature, 2013, 494, 336.

48 T. I. Lee, S. H. Lee, Y.-D. Kim, W. S. Jang, J. Y. Oh, H. K. Baik, C. Stampfl, A. Soon and J. M. Myoung, Nano Lett., 2012, 12, 68.

49 G.-Y. Huang, C.-Y. Wang and J.-T. Wang, Comput. Phys. Commun., 2012, 183, 1749.

50 Z. Wang, M. Zhao, X. Wang, Y. Xi, X. He, X. Liu and S. Yan, Phys. Chem. Chem. Phys., 2012, 14, 15693. 